\title{
Psychogenic stridor: an overlooked cause of acute stridor in the acute medical unit?
}

\author{
Authors: Hnin Zaw, ${ }^{\mathrm{A}}$ Tha Htet $\mathrm{Nyi}^{\mathrm{A}}$ and Win Win Aye ${ }^{\mathrm{A}}$
}

\section{Introduction}

Acute onset stridor is an alarming presentation for acute physicians, leading to high suspicion of organic pathology with immediate management directed towards the suspected diagnosis. One of the uncommon and overlooked causes of stridor in younger people is a vocal cord dysfunction, also called psychogenic stridor. It is often underappreciated and misdiagnosed in clinical practice, leading to overtreatment with unnecessary medications and development of significant side effects. A couple of those presentations were encountered in our acute medical unit and how they were managed is discussed here.

\section{Case presentations}

\section{Case 1}

A 21-year-old woman was admitted with acute onset stridor following a 3-day history of sore throat and failure to respond to initial general practitioner management with oral steroid and salbutamol inhaler. She had a background history of anaphylaxis to nuts, allergy to penicillin and bilateral tonsillectomy.

On examination she was mildly tachycardic, tachypnoeic and pyrexic, but normotensive and had no desaturation with predominately inspiratory stridor.

She reported similar features when she had had an anaphylaxis reaction in the past, but denied eating nuts/nut products and taking over-the-counter medications. She was treated for a possible anaphylaxis reaction by giving a stat dose of intramuscular adrenaline, injection of hydrocortisone, antihistamine, salbutamol nebuliser and adrenaline nebuliser. Her symptoms failed to improve and she became more tachycardic with chest discomfort. Electrocardiography (ECG) showed sinus tachycardia (130 beats per minute) with no ischaemic changes.

An urgent ear, nose and throat (ENT) assessment with flexible nasendoscopy excluded organic pathology. Arterial blood gases, routine blood tests and chest X-ray were unremarkable, as was her peak flow meter reading. A diagnosis of vocal cord dysfunction with viral illness was made. She improved with supportive management.

Authors: ${ }^{\mathrm{A}}$ Norfolk and Norwich University Hospital, Norwich, UK

\section{Case 2}

A 24-year-old man, generally fit and well, was admitted with a 1-day history of dry cough and intermittent stridor. He was not in distress but observations showed tachycardia, and saturation was $98 \%$ on $1 \mathrm{~L}$ oxygen. He was able to complete a sentence, but there was obvious stridor. Chest examination revealed good air entry with no wheeze. Full otolaryngological examination by ENT was normal. The chest X-ray, ECG and blood tests were unremarkable.

Due to his stridor, a stat dose of adrenaline nebuliser was given but reactionary tachycardia and chest discomfort were observed, which later settled on stopping the treatment. After having further ENT review for ongoing intermittent stridor, he was diagnosed with a dysfunctional vocal cord. He was reassured and discharged.

\section{Discussion}

Psychogenic stridor is sometimes difficult to treat as it is misdiagnosed and overlooked. Thorough history taking, physical examination and direct visualisation of the upper airway are crucial to rule out the organic causes. The treatment for psychogenic stridor is supportive management with reassurance.

\section{Conclusion}

There have been previous case reports in the literature where vocal cord dysfunction was mistakenly treated as asthma. Our two cases add to the growing evidence of the importance of early recognition and correct diagnosis of psychogenic stridor so as to prevent significant iatrogenic complications.

\section{Conflicts of interest}

None declared.

\section{References}

1 Deckert J, Deckert L. Vocal cord dysfunction. Am Fam Physician 2010;81:156-9.

2 Kenn K, Balkissoon R. Vocal cord dysfunction: what do we know? Eur Respir J 2011;37:194-200.

3 Wareing MJ, Mitchell D. Psychogenic stridor: diagnosis and management. J Accid Emerg Med 1997;14:330-2. 